\title{
LA ETIMOLOGÍA Y LA TRADUCCIÓN COMO RECURSOS EN LA ENSEÑANZA DE LENGUAS EXTRANJERAS
}

\author{
Cristina Martínez Fraile \\ Universidad de Sevilla
}

\begin{abstract}
The main aim is to show that it may obtain better results if the student connects his previous knowledges and new information. This article tries to demostrate how etymological information and traduction, as mnemonic techniques, can help students and teachers in Second Language Adquisition (SLA).
\end{abstract}

\section{INTRODUCCIÓN}

Cada día se inculca más que los europeos vivimos en una comunidad, la Unión Europea y de la necesidad de aprender lenguas. Por otro lado, las lenguas propias de cada país suponen un obstáculo en el propósito de formar una verdadera comunidad y para superar esta situación la traducción se presenta como una de las principales soluciones.

Sin embargo, la cuestión cambia mucho cuando se habla de "traducir" para enseñar una lengua extranjera. Durante los años setenta los métodos directos desacreditaban la traducción y la lengua materna como recursos para aprender una lengua nueva. No obstante, en este artículo se destacan todos los aspectos positivos que contiene la lengua materna en el proceso de aprendizaje de segundas y terceras lenguas. Es una postura que está tomando relevancia, sobre todo por la paulatina pero inminente implantación de los colegios bilingües.

\section{UN EJEMPLO PRÁCTICO Y SUS CONSECUENCIAS}

La mayoría de los estudiantes al empezar a aprender una lengua nueva comienzan traduciendo. Para demostrarlo en primer lugar se propone partir de un ejercicio práctico. Se trataba de 'entender' un texto en danés a pesar de que ningún asistente tenía nociones de la lengua danesa. Esta prueba fue llevada a cabo durante una ponencia en la que el público partía ya con unos conocimientos de alemán e inglés y español como lengua materna.

El objetivo de la prueba consistía en enseñar a los estudiantes cómo conectar sus conocimientos previos con la nueva información que debían aprender, concretamente con el vocabulario nuevo. Con ello se demostraría a los estudiantes que cuando se empieza a aprender una lengua totalmente desconocida siempre se buscan puntos de unión con los conocimientos anteriores de otras lenguas y que irremediablemente se acaba traduciendo. 
El ejercicio constaba de tres fases:

Fase 1. Presentación del texto en danés para extraer las primeras impresiones

Børn og fjernsyn

En amerikansk profesor i psykologi har beskǣftiget sig med, hvordan fiernsynet virker pa mindre børn. Hans undersøgelse drejer sig om børn med et meget stort fjernsynsforbrug. Hos dem udvikler sprogevnen sig kun langsomt, fordi de sidder passivt foran billedskǣrmen uden at deltage i samtaler med deres forǣldre, ogsa nar disse sidder ved siden af dem ${ }^{1}$. (Faerch, 1999: 17)

En esta primera fase todos los asistentes fueron incitados a leer y comprender "algo" del texto. Se comprobó que la cantidad de palabras reconocidas dependía de la lengua materna que poseían y del dominio de segundas y terceras lenguas. Aquellos que tenían como lengua materna el español y conocimientos de inglés y alemán reconocieron de inmediato las siguientes palabras que están en negrita.

Børn og fjernsyn

En amerikansk profesor i psykologi har beskæ̋ftiget sig med, hvordan fiernsynet virker pa mindre brøn. Hans undersøgelse drejer sig om børn med et meget stort fjernsynsforbrug. Hos dem udvikler sprogevnen sig kun langsomt, fordi de sidder passivt foran billedskæ̋rmen uden at deltage i samtaler med deres foræ̈ldre, ogsa nar disse sidder ved siden af dem.

Fase 2. Asociaciones y segundas impresiones

Había palabras dentro de este texto que no se reconocían tan fácilmente como las otras, pero les se les presentó algunas asociaciones entre palabras que no les 'sonaban' de nada y otras que sí conocían:

Fjernsynet - Fernsehen; Børn - ing. Born / dt. Geboren - niño; Virker - dt. Wirkung; Mindre - dt. Minder; Drejer - dt. Drehen; Sig - dt. Sich; Undersøgelse - dt. Untersuchen; Om - dt. Um; Udvikler - dt. Entwickelte; Billedskæ̋rmen - dt. Bildschirm; forǣldre >dt. Vater; af - ing. Of; dem - ing. Them

\footnotetext{
${ }^{1}$ Texto tomado de C. Fæ̋rch (1999).
} 
Con la ayuda de este tipo de asociaciones la comprensión del texto cambió algo más, tal y como refirieron todos los participantes.

\section{Børn og fjernsyn}

En amerikansk profesor i psykologi har beskǣftiget sig med, hvordan fiernsynet virker pa mindre børn. Hans undersøgelse drejer sig om børn med et meget stort fjernsynsforbrug. Hos dem udvikler sprogevnen sig kun langsomt, fordi de sidder passivt foran billedskǣrmen uden at deltage $\mathrm{i}$ samtaler med deres forǣldre, ogsa nar disse sidder ved siden af dem.

\section{Fase 3.Comprobación}

Se propuso de nuevo volver a leer el texto, aproximadamente cuarenta minutos después. Todos comprobaron cómo eran capaces de entender mucho más del texto que en la primera fase.

De este tipo de ejercicio se extrajeron una serie de conclusiones. Todos los asistentes coincidieron en haber experimentado lo mismo durante el proceso de reconocimiento:

a) Ante un estímulo desconocido (texto) todos intentamos comprender algo, reconocer palabras y relacionarlas con algunas de las que ya conocemos, tanto por nuestra lengua materna como por segundas o terceras lenguas.

b) Se produce un tipo de procedimiento mental llamado transferencia que viene dado porque las palabras, inicialmente 'desconocidas', comparten características fonéticas y gráficas con algunas que ya se conocen.

c) Es posible establecer relaciones entre palabras de distintas lenguas porque existe, además de ciertos procesos psicolingüísticos, un estrato común entre las lenguas europeas, como el indoeuropeo y distintas familias lingüísticas.

d) En este proceso tiene un papel fundamental la etimología.

Este mismo ejercicio se propuso para los profesores de cualquier lengua extranjera $y$, sobre todo, dirigido a un grupo de estudiantes de nivel inicial.

\section{UN CASO CONCRETO: APRENDER VOCABUlaRIO ALEMÁN UTILIZANDO LA ETIMOLOGÍA}

La motivación para centrarme en el léxico alemán nace en primer lugar de mi experiencia como estudiante de Filología Alemana. La mayoría de los estudiantes coinciden en que cuando se trata de aprender alemán, el vocabulario es uno de los principales obstáculos que cuesta superar. No ocurre lo mismo con lenguas como el francés o el italiano porque 'las palabras se parecen al español'. 
De estas observaciones se deduce que la primera estrategia, casi innata, de aquel estudiante que se dispone aprender una nueva lengua es aprender desde su misma experiencia lingüística.

A este estudiante se le presentan dos problemas:

$1^{\circ}$ ) La gran mayoría de los métodos se han centrado en la transmisión de contenidos gramaticales y de aspectos morfosintácticos.

$2^{\circ}$ ) La enseñanza del léxico ha sido una de las áreas más ignoradas en la historia de la enseñanza de lenguas extranjeras. Precisamente, por el escaso tratamiento didáctico en clase, aprender vocabulario es y sigue siendo la parte más áspera para el estudiante.

Del mismo título he tomado las palabras a partir de las cuales iré desarrollando el artículo: "La etimología y la traducción como recursos "atrevidos"en la enseñanza de lenguas extranjeras"

\subsection{La etimología}

La etimología se define en este contexto como recurso para enseñar vocabulario en una clase de lengua extranjera. Rask (1992: 40) en su obra Von der Etymologie überhaupt la presenta de este modo tan metafórico: "Die Etymologie ist nun wie der Geist in dieser toten Masse, das Banda, welches die einzelnen Teile zu einer ununterbrochenen Kette zusammenfügt und verbindet.

La masa muerta a la que el autor se refiere se identificaría con el conocimiento lingüístico, tal y como sugiere Rask. De este modo, es posible imaginar el cerebro de un alumno en el que se acumulan muchas palabras de las distintas lenguas: la materna, otras segundas y terceras lenguas que aprende. Y todas las palabras se encuentran en el cerebro como si perteneciesen a sistemas separados.

El hecho de que cada lengua se considere un sistema cerrado y separado de todas las demás lenguas obliga a reflexionar sobre el proceso de aprendizaje de una lengua nueva y desconocida. En este caso el alumno siempre tendrá la sensación de "empezar desde cero" con cada lengua y de no poder alcanzar la perfección en ningún idioma, una perfección que por otro lado es un tanto utópica sobre todo en un primer nivel. De este modo, sólo se consigue romper con la motivación del alumno.

No obstante, en la labor de enseñanza, es necesario estimular a los alumnos haciéndoles ver que existen lazos de unión entre las lenguas, circunstancia de la que se puede sacar provecho. Se trata de concienciarlos para que descubran 'lo conocido en aquello que parecía desconocido'.

La intención de buscar 'lo conocido', los lazos de unión, las afinidades y no las diferencias son valores que están presentes en el día a día. La Unión Europea como institución tiene como propósito crear una unidad política, una unidad económica y por qué no una unidad lingüística. Esto no significa la desaparición de todas las lenguas y la hegemonía de una sobre las demás, sino que el espíritu de la unidad comparte además el respeto por la identidad de cada pueblo. 
La etimología contribuye a superar los obstáculos y las barreras lingüísticas. Es precisamente esa ciencia auxiliar que ayuda a crear lazos entre las palabras de las distintas lenguas presentándose como la 'banda' que intenta, como decía Sweet, que un grupo de viejos amigos o de primos se reencuentren. Sobre todo en el siglo XXI el sentimiento fundado en la pertenencia a una gran familia lingüística europea adquiere gran valor.

Mastering the vocabulary of most European languages means simply learning to recognize a number of old friends under slight disguises, and making certain effort to learn a residue of irrecognizable words, which, however, offer less difficulty than they otherwise would through being imbedded in a context of familiar words. The higher vocabulary of science, art, and abstract thought hardly requires to be learnt at all; for it so consists either of Latin and Greek terms common to most European languages or translations of them. (SWEET, 1899/1972:664-65 en ODLIN, 1989).

\subsection{La etimología en la enseñanza de lenguas extranjeras}

En la enseñanza de las lenguas extranjeras, según mi punto de vista y la de otros muchos, es preciso apreciar todo el parentesco que las lenguas comparten. Autores como Robert Ilson y Herbert D. Pierson empezaron a barajar la posibilidad de utilizar la etimología en clase a finales de los años ochenta. En esta época ambos publican en la revista ELT Journal dos artículos que hacen concretamente referencia al uso de la etimología en clase: el primero de ellos fue "Etymological information: can it helps our students?", de Robert Ilson (1983) y el segundo artículo "Using etymology in the classroom", de Herbert D. Pierson (1989).

Pierson se manifiesta a favor del valor cualitativo de la etimología aplicada a la didáctica de lenguas extranjeras. De este modo, se evitaría la seca memorización de vocabulario y se fomentaría el proceso asociativo entre palabras de distintas lenguas. Este proceso favorecería la retención a más largo plazo.

I believe a meaningful approach to etymology in second-language learning, as opposed to rote memorization of words, prefixes, suffixes and roots, can offer intermediate/advanced second-language students both practical and theoretical linguistic knowledge congenial to a more permanent retention of words and concepts. (Pierson, 1989).

Por tanto, la etimología es un recurso del que se beneficiarían tanto el profesor como el alumno:

a) La etimología puede ser un buen material para los profesores de segundas lenguas en tanto que, al mostrar al alumno la relación de dos palabras bajo una explicación lógica, se consigue potenciar un aprendizaje significativo. 
Although etymology stands at the periphery of second-language teacher education programmes, etymological training could benefit second-language instruction. The teacher and student, by becoming serious amateur etymologists, would find themselves more sensitive to the meaning of words and their relationships with other words from both history and other languages. The knowledge of these word relationships could contribute to what educational psychologist call meaningful learning (Ausable, 1968), a quality of learning which is related to prior learning, and thus is more likely to be retained and generalized to other learning. (Pierson, 1989: 58).

b) Para el estudiante de lenguas extranjeras la etimología se presenta como un recurso complementario, ya que el objetivo no consiste en aprender etimologías. El alumno solamente se beneficiaría de las asociaciones propuestas por el profesor y acompañadas, en ciertos casos, de explicaciones etimológicas.

De este modo se conseguiría potenciar el desarrollo de las competencias:

a) Receptiva: se favorece la lectura y comprensión de textos.

El reconocimiento de las palabras se resuelve mediante la aplicación de estrategias basadas en la asociación de palabras en función de las similitudes gráficas y fonéticas entre palabras.

b) La competencia de almacenamiento. Se potencia una memorización a más largo plazo al relacionarse palabras desconocidas con aquellas que ya se conocen.

Hieraus fließt ein anderer, nicht unbedeutender praktischer Nutzen der Etymologie oder der Spracherklärung für die, welche fremde Sprachen erlernen. Die Wörter, die sie zu erinnern haben, werde lange nicht so viele oder so beschwerlich für das Gedächtnis, wenn man ihre Verwandtschaft und Ableitung sorgfältig beachtet. Es sind dann nur die Stammwörter, die einfach behalten werden müssen, (...). Aber noch weitaus gröBer wird der Vorteil, wenn die fremde Sprache mit der Muttersprache verwandt ist oder wenn man mehrere Sprache miteinender verwandte fremde Sprache lernt. Wenn man sich ein für allemal die Übergänge gemerkt hat, dann ist man selbst imstande, sich die Wörter zu bilden, und es kostet nicht die halbe Mühe, sie dann in zwei oder mehr Spachen zu erinnern, die es ohne dies Mittel kosten würde, sie in einer zu erinnern. (Rask, 1992: 53).

Las investigaciones más recientes en esta línea han producido trabajos de gran interés en relación con las ideas propuestas en este artículo. Se trata concretamente del proyecto 
EuroComRom-Die sieben Siebe de Horst G. Klein y Tilbert D. Stegmann y del proyecto Deutsch nach Englisch junto a los que mi estudio podría encuadrarse.

El primero de los proyectos apuesta por la enseñanza de terceras lenguas a partir de los conocimientos previos (como supone la lengua materna u otras segundas lenguas).

Das Bedeutet: Sprachbewussheit durch Vergleichen von L1 und L2 und Besprechen der Wahrnehmungen fördern:

Beispiele: Was ist ähnlich? Wo kann man anknüpfen? Was ist ganz anders? Wo gibt es "Fallen" (Interferenzen)? (...). Je enger die Muttersprache und die erste Fremdsprache sprachtypologisch verwandt sind, je mehr Internationalismen und Lehnwörter aus der neuen Sprache sich in der Muttersprache vorfinden, desto mehr Anknüpfungspunkte und Transfermöglischkeiten wir man z.B. bei den Sprachsystemen (Gramatik; Wortschatz; Aussprache; Rechtschreibung) herstellen können. (Hufeisen, 1993: 23).

Klein en su obra EuroComRom. Die sieben Siebe (2000) pone en contacto algunas lenguas románicas. Para ello presenta las afinidades gráficas y fonéticas de sus respectivos léxicos gracias a que pertenecen a una misma familia lingüística.

La cuestión se complica más cuando se trata de poner en relación dos lenguas pertenecientes a familias lingüísticas distintas como el alemán, de la familia germánica, y el español, una lengua románica. Sin embargo, puede resolverse de la siguiente forma tomando la etimología como referencia:

Artz (médico) < gr. archiatros > pediatra, -atra

Bier (cerveza) $<$ lat. bibere $>$ beber, biberón

Insel (isla) $<$ lat. insula $>$ insula, isla, península

Fall (caída) $<$ lat. fallere $>$ fallecer, flácido

Lied (canción) < lat. Laus > laudatorio

En estos casos propuestos no se da una coincidencia de significados. No obstante, simplemente con las afinidades semánticas parciales se ofrece un recurso para recordar la palabra a más largo plazo.

\subsection{Opiniones divergentes entorno a la traducción como recurso}

En el título del artículo se presentaba la etimología y la traducción como recursos para la enseñanza de lenguas extranjeras. Aunque la traducción es un ejercicio muy complejo, en 
este contexto se entiende como la utilización de la lengua materna y de otras lenguas para comprender una lengua nueva.

Se propone la traducción como:

a) Medio y no como fin. Se trata de una traducción como recurso para establecer la comunicación y de la que se puede aprender. No se trata realizar una traducción como actividad profesional en la que se dispone de una serie de diccionarios especializados, incluso tampoco de una simple traducción en la que se utiliza un diccionario.

b) Recurso en la enseñanza de lenguas extranjeras, incluso puede servir para cualquier persona que viaja a un país extranjero o toma un texto en otra lengua que desconoce.

c) Una traducción 'interlingüística' en la que participan todas las lenguas que el alumno conoce y todos los conocimientos previos. El alumno pone en funcionamiento todos los recursos posibles para comprender el texto. Por ejemplo, en el texto inicial la palabra "passivt" se podía comprender desde el español y "beskaeftiget" a través del alemán.

Los ejemplos propuestos en el apartado anterior son sólo una muestra que ilustra cómo las comparaciones entre la nueva lengua y los conocimientos previos de otras lenguas mejoran la capacidad de reconocimiento, comprensión y almacenamiento.

Autores como Dulay y Burt (Byran, 2000: 663) dudan de la legitimidad de este tipo de estrategias. Para ellos no existen equivalencias exactas entre las lenguas porque las palabras en cada lengua han ido adquiriendo matices que determinan su significado. La estrategia de comparar lenguas no sólo es arriesgada sino también perjudicial para el aprendizaje, dado que sólo producen interferencias negativas y dificultan el proceso.

Y realmente, no se puede negar que existan las interferencias y falsos amigos cuando se comparan lenguas. Pero tampoco puede evitarse que siempre existan las comparaciones casi de una forma instintiva y automática.

Sobre todo cuando empezamos a aprender una lengua nueva el profesor insiste siempre con frases como "no traduzcáis", "hay que pensar en alemán". Incluso la experiencia demuestra al estudiante que, pese a su intento, casi de forma secreta, sigue pensando en su lengua materna a qué equivale lo que se está diciendo en la lengua desconocida. Se procura que el alumno aprenda la segunda lengua igual que lo hizo como adquirió la primera. No obstante no se puede eliminar la experiencia lingüística con la que ya partimos, sobre todo en los primeros niveles.

También en los métodos actuales se ha dado un giro al problema de los errores y al de los falsos amigos a los que tanto se aferran los detractores de esta postura. Hoy día el error no está considerado como un elemento negativo sino como un comportamiento natural e inevitable en el proceso de aprendizaje. Si antaño el error era una falta grave que a veces desanimaba al alumno a seguir avanzando, hoy los errores se conciben como casos de los que se pueden aprender. Klein resuelve así el problema de los falsos amigos: 
«Me voy a confundir si aprendo una lengua parecida. Tengo miedo a mezclar». (...) Por lo que respecta a la mezcla de lenguas es necesario ser siempre consciente de la gran ventaja que representa reconocer palabras inmediatamente por su parecido con las de otras lenguas y poderlas recordar sin tener que hacer un esfuerzo de aprendizaje.(...). Una vez más es necesario subrayar que el hecho de que al comienzo del aprendizaje de una lengua sea posible valerse de palabras de otra lengua relacionada no es de ningún modo un obstáculo, sino un gran alivio ${ }^{2}$.

Resulta en este sentido más positivo aprovecharse del parecido entre lenguas para entender al menos alguna información en la lengua extranjera y se acaba desterrando el miedo de equivocarse a la hora de entablar la comunicación.

\section{Conclusiones}

No puede negarse, sobre todo en los primeros niveles de comprensión, que la información que se recibe se intenta trasladar a nuestra lengua materna o relacionarla con otras lenguas que ya conocemos.

Para finalizar destacaré cuatro ideas principales:

a) Cuando uno se dispone a aprender una lengua no es 'tabula rasa' y que parte ya con unas estructuras lingüísticas y un léxico que, de cualquier modo, condicionan la adquisición y el aprendizaje de una lengua nueva.

b) Si no se puede eliminar el estrato que ya se ha adquirido, no hay que hacer un esfuerzo por eliminarlo sino por aprovecharlo.

c) El hecho de utilizar la etimología para establecer la relación no es en principio tarea del alumno, sino un recurso para que el profesor le desvele al alumno ciertas asociaciones entre palabras, asociaciones que favorezcan su memorización.

d) Para aprender una lengua extranjera traducir no es imprescindible, pero a veces 'traducir relacionando' es necesario, muy útil y rentable.

\section{REFERENCIAS BIBLIOGRÁFICAS}

BAUSCH, R., Handbuch Fremdsprachenunterricht, Tübingen - Basel, Francke, 1995.

BYRAN, M. (ed.), Routledge Encyclopedia of Language Teaching and Learning, New York: London and New York, 2000.

BOHN, R., Probleme der Wortschatzarbeit, Kassel, DIFF/Goethe Institut, 2000.

DUDEN ETYMOLOGIE, Herkunftswörterbuch der deutschen Sprache, Mannheim, Dudenverlag, 1989.

\footnotetext{
${ }^{2}$ Traducción aproximada de un fragmento de H. Klein, H./Stegmann, T., EuroComRom. Die sieben Siebe, Aachen: Shaker, pp. 18.
} 
F/ERCH, C., Learner language and language learning, England, Multilingual Matters LTD, 1984.

F $\overrightarrow{E R C H}, \mathrm{C}$. , One Learner-two Languages: Investigating Types of Interlanguage Knowledge, University of Copenhagen - Gabriele Kasper, University of Aarhus, 1999.

HUFEISEN, B. / NEUNER, G., Mehrsprachigskeitskonzept: Tertiärsprachenlernen Deutsch nach Englisch Europarat, Goethe Institut, 1993.

ILSON, R., "Etymological information: can it help our students?", ELT Journal, vol. $37 / 1,1983$, pp. $57-58$.

KLEIN, H. / STEGMANN, T., EuroComRom. Die sieben Siebe, Aachen, Shaker, 2000.

KLUGE, F., Etymologisches Wörterbuch der deutschen Sprache, Berlin, de Gruyter, 1989.

KYTZLER, B. / REDEMUND, L., Unser täglisches Latein, Mainz am Rhein, Philipp von Zabern, (1992), 2001.

RASK, K., Von der Etymologie überhaupt? Eine Einleitung in die Sprachvergleichung, Tübingen, de Gruyter, 1992.

MACHT, K. / STEINER, F., Erfolgsfaktoren des Vokabellernens, Augsburg, Universität Augsburg, 1983.

MANCHÓN, R., "Un acercamiento psicolingüístico al fenómeno de la transferencia en el aprendizaje y uso de segundas lenguas”, Estudios de Lingüística, Alicante, Universidad de Alicante, 2001.

MEISSNER, F.-J. / REINFRIED, M., "Von induktiven zum konstruktiven Lehr- und Lernparadigma. Methodische Folgerungen aus der mehrsprachichkeitsdidaktischen Forschung", en: Meissner, F.-J., Bausteine für einen neokommunikativen französischunterricht, Tübingen, Narr, 1998.

QUETZ, J., Neue Sprachen lehren und lernen. Fremdsprachenunterricht in der Weiterbildung, Bielefeld, DIE, 2002.

ODLIN, T., Language Transfer. Cross-linguistic influence in language learning, Cambridge, Cambridge University Press, 1989.

SEGURA, S., Diccionario etimológico latino-español, Madrid, Ediciones Generales Anaya, 1985.

SIGUÁN, M., La Europa de las Lenguas, Madrid, Alianza, 1996.

SWAN, M., "The influence of the mother tongue on second language vocabulary acquisition and use", en: Schmitt, N. / Mccarthy, M., Vocabulary: Description Acquisition and Pedagogy, Cambridge, University Press, 1997.

WALDE, A., Lateinisches Etymologisches Wörterbuch, Heidelberg, Carl Winter,1956. 